\title{
¡QUE VIVA CHANGÓ! MÚSICA Y RELIGIOSIDAD AFROCUBANAS EN EL PERÚ EN TRES DÉCADAS (1980-2010)
}

\section{¿QUE VIVA CHANGÓ! MUSIC AND AFRO-CUBAN RELIGIOSITY IN PERU IN THREE DECADES (1980-2010)}

\author{
Juan Manuel Saldívar Arellano* \\ Juan Pablo Anticona Cebrián**
}

RESUMEN

Las migraciones de cubanos al Perú ha sido un tema poco estudiado; sin embargo, poner atención al establecimiento de las oleadas migratorias de cubanos que se han anclado en el país a partir de un conflicto político gestado en Cuba en 1980, lleva a describir fenómenos periféricos, tales como, la influencia musical y las tradiciones religiosas afrocubanas como

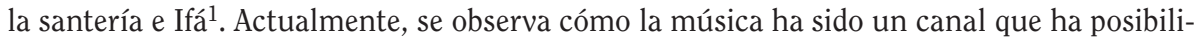
tado la influencia religiosa en el país a partir del arribo de agrupaciones musicales cubanas a los principales escenarios de Lima, la popularización de la "salsa" en restaurantes, discotecas, bares cubanos y la formación de casas templo ${ }^{2}$ en la ciudad. Este artículo propone una aproximación en torno a los antecedentes de cubanos en el Perú, considerando la influencia de la música como un factor determinante para la popularización de las tradiciones religiosas como filtraciones culturales transnacionales entre personas, países y lugares.

\section{PALABRAS CLAVE:PERÚ*MÚSICA * RELIGIÓN SOCIOLOGÍA CULTURAL* SANTERÍA}

\section{ABSTRACT}

The cuban migration to Peru has been an issue that has not been explored with profundity however, playing certain attention to the establishment of the migratory waves of cubans that have anchored in the country since the political conflict origined in Cuba in 1980. This will leads to describe peripheral phenomena, such as musical influences and afrocuban religious tradition like santeria and Ifá. We can observe how music has been a

\footnotetext{
* Universidad de Tarapacá, Chile. jsaldivar@uta.cl

** Investigador independiente. janticona@gmail.com

1 Sistema religioso procedente del grupo yoruba, se estableció en Cuba a partir del tráfico de esclavos africanos en tiempos de la Colonia.

2 Espacio donde se desarrollan las prácticas religiosas de la santería e Ifá, regularmente se utilizan casas particulares con lugares dedicados al culto de las divinidades llamadas orichas.
} 
channel that has allowed religious influence in the country since the arrival of cuban musical groups into the mainstream of Lima. The popularity of "salsa" in restaurants, night clubs, cuban bars and the creation of temple houses in the city. This article proposes an approach of cuban background in Peru; considering the influence of music as a determining factor in the popularization of religious traditions as transnational cultural leaks between the people, countries and places.

KEYWORDS: PERU* MUSIC * RELIGION* CULTURAL SOCIOLOGY * SANTERIA

\section{INTRODUCCIÓN}

El primer antecedente de cubanos en el Perú apunta a Leoncio Prado Gutiérrez, hijo del ex-presidente peruano Mariano Ignacio Prado,considerado héroe del Perú, quién viajó a Cuba en 1873 para unirse a las filas del ejército español. Finalizada las luchas con la victoria española y teniendo en cuenta la declaratoria de guerra de Chile al Perú, Leoncio Prado regresa a Lima con su compañero cubano de armas Juan Luis Pacheco de Céspedes "El cubano" (1853-1895), primer cubano del que existen referencias en el Perú.Al ser Cuba uno de los países americanos pequeños con independencia más reciente (1902), sus habitantes en su mayoría emigraban hacia el Caribe (Puerto Rico, Haití, Jamaica), al Norte (Estados Unidos y México) o a Europa (Francia, Italia), incluso a África; no obstante, los ciudadanos cubanos raramente emigraban al Sur.

El hecho fundamental que vincularía a Cuba con el Perú, a nivel político, fue la revoluciónencabezada por Fidel Castro Ruz y que puso en el mapa político al socialismo. En efecto, hacia 1959, el socialismo se percibía como una ideología que colocaba al pueblo por encima de las cosas, siendo muy atractiva por las mayorías populares. En el Perú, la intelectualidad creó el social progresismo, que aspiraba a transformar la sociedad peruana al estilo de Cuba, pero sin fusilamientos ni ejecuciones masivas.Juan Velasco Alvarado, presidente del Perú durante 1968-1975, aceptó la sugerencia de sus asesores que, siendo el gobierno revolucionario de las fuerzas armadas de tendencia social progresista, podría resolver el problema del campesinado y del pueblo, en general.
Esta influencia no progresó desde la esfera política, pero si desde otros ángulos socioculturales, como la música y las tradiciones religiosas de origen cubano. A partir de 1980, las migraciones de cubanos al Perú se hicieron notar en oleadas por etapas. Otras se originaron a partir de las referencias de amigos $y$ familiares que residían en Cuba, externando las posibilidades por salir de la isla, fue así que llegaron invitados cubanos a Lima. Se podrían citar dos etapas migratorias de cubanos al Perú, la primera que va de 1980 al 2000 y la segunda del 2001 al 2010. Algunas posibilidades que les permitieron a los cubanos salir del país fueron la música, la medicina y el deporte, lo cual también les permitió mantener vínculos entre el lugar de origen y el de destino. En este sentido, las contribuciones de cubanos al Perú van más allá de un anclaje, pues es a partir de estos movimientos que las tradiciones culturales $y$ religiosas se popularizan en Lima, así como también en otras ciudades de provincia como Arequipa, Cusco y Trujillo. No solo la religión como tradición cultural del acervo identitario cubano se difundió en el Perú, la música también ha sido parte de un desarrollo cultural de característica caribeña, aunque ya se tenía referencia de los ritmos cubanos en el país, la llegada de las comunidades migrantes fortaleció los lazos rítmicos y enalteció, popularizando las melodías del pasado-presente cubano. Actualmente, se observa cómo la industria musical de origen cubano ha crecido, sobre todo en la capital del país, a partir del establecimiento de espacios de ocio como restaurantes, discotecas $y$ bares estilo cubano que se manifiestanen las avenidas principales de Miraflores, San Borja, Chorrillos y otros distritos limeños. 
La música de origen cubano en el Perú proliferó a partir de la diversidad de ritmos, siendo el género musical conocido como "salsa" el que se estableció en los imaginarios culturales de los peruanos, quienes lo adoptaron como parte de un acervo identitario caribeño. Si bien es cierto "hasta antes de 1959 Cuba era el país latinoamericano que más contribuyó en el enriquecimiento de los repertorios ofrecidos" (Yudice, 1999:231), pues algunos países geográficamente cercanos como Estados Unidos, México y otros de Centroamérica, adoptaron desde épocas tempranas (a partir de los años 30 aproximadamente) sus géneros musicales para re-contextualizar los propios (Figueroa, 2002; Juárez, 2011 y Knauer, 2001). Sin embargo, para el caso de Sudamérica podría afirmarse que estos géneros musicales llegaron "tarde", es decir, a partir de los años 60 con la influencia de la "salsa" como género musical innovador en el continente. Las industrias culturales del cine y la música se valieron de esta influencia rítmica haciendo uso de múltiples "estrofas" para representar las producciones cinematográficas de mediados de siglo pasado (Podalsky, 1999). Ritmos como la guaracha, el danzón, la rumba, el son, habanera, la música de casino y sus modificaciones al estilo puertorriqueño y estadounidense conocido como "salsa", fueron algunos de los géneros musicales que aparecieron en los principales escenarios latinoamericanos después de los años 30.

\section{ANTECEDENTES DE LA MÚSICA CUBANA EN EL PERÚ (1930-1970. PRIMERA ETAPA MUSICAL)}

Al final del siglo xix en Cuba, las principales orquestas de música reconocidas en el exterior eran "Concho de oro", "Flor de Cuba", "Failde", "Perico Rojas" o "Felipe Valdez", así como, la danza y la contradanza. En 1879 es creado el primer danzón "Alturas de Simpson" por Miguel Failde de la "Orquesta Failde". De otro lado, el bolero, como género romántico apareció aproximadamente en 1885 , la orquesta típica fue reemplazada por la orquesta "Charanga" alrededor de 1900. En este caso, las orquestas serán de sonido más fuerte con la introducción de la flauta y los timbales. Las más famosas son las de "Torroella", "Tata Alfonso" y "Romeu". La orquesta "Torroella" grabó seis temas en el cilindro de Edison, la orquesta"Tata Alfonso" grabó seis danzones para la Columbia Records, en 1918. Sin embargo, la orquesta "Romeu"fue la que trascendió musicalmente entre los años de 1910-1990, grabando más de cien temasy continuando después de la muerte de su fundador Antonio María Romeu (18761955), incluso después del fallecimiento de su afamado continuador, Barbarito Diez, "La voz de oro del danzón".El dúo "Montes y Manrique" grabó los primeros temas de la "Guardia vieja" (1885-1900) en New York, para la Columbia Records, entre 1905-1911. Los primeros grupos cubanos empezaban a sonar en Europa a mediados de los años 20,pero gracias al éxito "El manicero" (1930) interpretado por Antonio Machín, fue que se abrieron muchas puertas para los intérpretes cubanos. Machín viajó a New York en 1930 y de allí pasó a Europa en 1936, donde formó su orquesta. Al parecer, la salida de grupos cubanos al exterior fue producto de su gran éxito en la isla, de la ubicación de esta y de las grabaciones que gustaron en el exterior.

De acuerdo a la evolución de la música cubana, Juárez Huet retoma los planteamientos de Podalsky (1999), quien argumenta cómo a partir del siglo xix"la música popular cubana se dio a conocer por medio de Los Bufos Habaneros, un grupo de teatro popular nacido en Cuba...que dio lugar a una serie de grupos cuyas presentaciones eran de tono paródico..." (2011:165). Si bien, el interés de la autora es matizar la influencia de la música cubana en México, asegura que la evolución de estos ritmos musicales generó una serie de diversidades sonoras a partir de la contradanza seguidas del danzón, la guaracha y el son cubano (Sevilla, 1998), mismas que fueron apreciadas en los principales escenarios de México, siendo el Carnaval de Veracruz uno de los espacios que los popularizó. Otros lugares de acogida que no solo hacían referencia a escenarios musicales, fueron los puertos en zonas marítimas, lugares como Veracruz, Mérida, Campeche y otros espacios cercanos a Cuba (Juárez, 2011). La misma autora alude al auge del cabaret en Cuba y sus representaciones musicales que ofrecían repertorios amplios de ritmos, algunos de estos 
vinculaban las tradiciones religiosas de la santería como parte de la sonoridad mostrada en los mismos. Matiza que el son cubano fue uno de los géneros que más se difundió fuera de Cuba, en países como Estados Unidos y México (Knauer, 2001).

En lo que refiere al Perú, al encontrase situado geográficamente a distancias considerables de Cuba, las conexiones musicales, así como, el auge pasivo de las industrias culturales en comparación con México, tardaron en incorporarse en el territorio peruano, por lo cual tuvieron que aparecer circunstancias vinculantes de carácter periférico como el auge marítimo entre Cuba y Perú a través del Puerto del Callao, lugar en el cual encallaban barcos cubanos y algunos de sus tripulantes hacían difusión de la música nacional cubana. De esta forma fue que "-la música de Cuba llega a través del Callao, se vinculó primero con los trabajadores negros que interceptaban los buques cubanos en el Callao, ellos fueron quienes mostraron la música cubana en el Perú" (José, entrevista, 10/11/2010).Otro aspecto a considerar fue el apogeo de los valses limeños de la "Guardia Nueva" representada por Felipe Pinglo, además de las variedades musicales manifestadas en los teatros, salones y callejones que expresaban los valses criollos, los foxtrots, los onesteps y el tango argentino que irrumpía el bagaje cultura limeño de la década de los años 20 y 30 .

Una de las primeras agrupaciones musicales que arribóal territorio peruano fue "Lecuona Cuban Boys" originariamente de Ernesto Lecuona, visitandoen varias ocasiones la ciudad de Lima entre 1939-1945. Durante los años 40, fueron varios los grupos que sonaron en Lima y provincias, por esa época, después de la muerte de Gardel (1935), los sonidos de procedencia cubana tuvieron popularidad en Lima $y$ algunas ciudades del país. Música de los "Lecuona Cuban Boys", la "Gloria Matancera", la "Sonora Matancera", "Celina y Reutilio", y "El trío Matamoros" sonaban en las emisoras peruanas. Posiblemente, esto favoreció la llegada de los "Lecuona" que venían trayendo como novedad los primeros mambos.Por otro lado, el conjunto "Sonora Matancera", formado en matanzas en 1924, lanzó el disco "La ola marina" a fines de 1944, presentando a Bienvenido Rosendo Granda Aguilera como nuevo intérprete solista. La "Ola marina", "Bamba alegre", "La vaca lechera", "Micaela" y otros, fueron grandes éxitos tanto en Lima como en provincias. Ante la fuerte demanda, el mercado musical se abrió no solamente con la aparición de músicos a escenarios limeños, sino también la mercantilización de su música a través de las colecciones en discos de carbón producidos hasta ese momento por Stinson o Pan-art.

Un aspecto a destacar es la música que aludía a las tradiciones religiosas cubanas, como:

$\diamond \quad$ "Cuarteto Caney" (1939-1940): "Mi
padrino me manda", "Un brujo en
Guanabacoa".
"Celina y Reutilio" (1948-1955): "A Santa
Bárbara", "San Lázaro", "A la caridad del
cobre", "A la virgen de las mercedes", "E1
Hijo de Elegguá".
Celia Cruz con la "Sonora Matancera"
(1950-1957): "Chango táveni", "Yembe
la roco", "Palo mayimbe", "Eleguá
quiere tambó", "Imoye", "Quede
Zaina", "Yemayá", "Para tú altar",
"OyemeAggayú", entre otras.

En los 40 y 50, la música con temática religiosa se reflejaba más en los géneros como: la rumba, el guaguancó, la columbia o el llamado "afro". Sin perjuicio que existe un ritmo determinado para el tema de la santería, que es el toque de los tambores de batá, los cuales ya se han utilizado también para generar sonidos populares, lo que ha causado una sonoridad autóctona a la música cubana en general. En este sentido, se afirma que los peruanos de la época, si bien es cierto, asimilaban con desconocimiento religioso la música cubana y sus letras, tomaban el tema religioso inmerso en ella como algo poco curioso o exótico, desconociendo el tema de la religión y no deseando profundizar mucho al respecto, considerándolo como ritmos que trataban de "la religiosidad de los africanos" en Cuba. 
Los grupos cubanos impactaron de una manera diferente en el Perú en los años 40, llegando a su primer apogeo en la década del 50 (1950-1959). Fue la"Sonora Matancera" que incluso después de 1948, adoptó un sonido más estilizado e internacional, los "Havana Cuban Boys", la "Orquesta de Dámaso Pérez Prado", el dúo "Los compadres" (Lorenzo Reyezuelo ahora con su hermano menor Reynaldo, "Rey Caney"), Guillermo Portabales, el trío "La Rosa", "los Guaracheros de Oriente",el trío "Servando Díaz", entre otros, se hicieron populares en las reuniones limeñas y de la costa de Perú. El tango bajaba en popularidad y la competencia ahora sería con el vals costeño que había entrado a los salones, ya que antes era considerado de estrato popular. Es curioso el hecho de que el vals criollo peruano siempre tuvo competencia, pues entre los años 10 y 20 compitió con los foxtrots, los onesteps;en los 30 , con el tango y el pasodoble; $y$ a partir de los 40, con la música cubana y mexicana, no llegando a conseguir el esplendor internacional que tanto mereció. La ranchera mexicana llegaba de la mano de Jorge Negrete y Pedro Infante; no obstante, en México se recibía la influencia de Cuba desde los años 40. Por ejemplo, los antecedentes de la música cubana en México son más extensos que en el Perú;Beny Moré había llevado el afro, las guarachas y el mambo junto con Pérez Prado. El cubano "Acerina" había llevado el danzón a este país, el cual lo acogió llegando al extremo de renovarlo (Chamorro, 1948; Flores y Escalante, 2006). Esta influencia conllevó a que surgieran también compositores mexicanos y puertorriqueños de bolero.

De acuerdo al triunfo de la revolución cubana y la consolidación del régimen castrista, muchas agrupaciones que habían logrado surgir en la isla y fuera de ella, emigraron a diversos países sin intenciones de retornar en una real diáspora. La "Sonora Matancera" y los "Lecuona Cuban Boys", por ejemplo, se establecieron en Nueva York;"Rolando La Serie", en Miami; Orlando Contreras, en Colombia; Dámaso Pérez Prado se quedó en México, igual que Consejo Valiente Robles "Acerina", el de "Acerina y su danzonera". En cambio, el dúo "Los compadres", por su filiación comunista y apoyo al gobierno permaneció en la isla. Con el bloqueo y aislamiento de Cuba al mundo por parte de los Estados Unidos aproximadamente en 1962, la música mexicana tomó el primer lugar de las preferencias en Perú. Se escuchaba Pedro Infante, que ya había muerto, José Alfredo Jiménez, Antonio Aguilar, Miguel Aceves Mejía y fundamentalmente, Javier Solís "el rey del bolero ranchero". Durante la década de los 60 , se erigió otro de los grandes músicos que se popularizó en el país, Armando Manzanero con sus temas románticos; sin embargo, el repertorio musical cubano fue desapareciendo.

Los géneros musicales cubanos, entre estos, la trova, hicieron eco en Perú de manera diezmada, llegados los años 70 la mayoría de agrupaciones que en su momento lograron un auge musical, retornaron a los escenarios peruanos con estilos diferentes a los antes conocidos, sobre todo recordando sus éxitos, incluyendo uno que otro tema nuevo. La "Sonora Matancera" comenzó a interpretar un nuevo género nacido en Nueva York conocido como "salsa". En Perú, la orquesta brasilera "Cuba Libre" traería nuevamente, a principios de los 70, los viejos éxitos de Cuba, así también la llegada del rock and roll, la nueva ola, la "salsa", el bolero cubano que cedería al final su sitial a la balada; manifestaban el descenso en la calidad musical, tanto de la interpretación como de la ejecución. El primer apogeo de la música cubana terminaría en Lima; sin embargo, la poca influencia de la música cubana en el país se mantuvo a partir de intérpretes locales como el argentino Freddy Roland, el chiclayano RullyRendo, Lucho Macedo y finalmente, Ricardo "el Mocho" Bustamante. Estas interpretaciones siguieron apareciendo hasta la década de los años 80 .

En el caso de México, la producción musical cubana favoreció en diversos sentidos, por un lado, la relación geográfica que permitía a los cubanos acercarse a México, por otro, la generación de músicos que se estableció en el país, aportando una serie de ritmos que fueron de acogida por otros intérpretes nacionales. Juárez Huet asegura que la influencia cubana en las industrias culturales mexicanas fue productiva a partir de tres aspectos "...el populismo de barriada, 
el género de las cabareteras y el género del amor familiar" (Monsiváis, 1998:1522, citado en Juárez, 2011:171). Además, en lo que se refiere a la música principalmente "tanto los músicos cubanos y mexicanos de la época como varias de las figuras clásicas con quienes compartieron escena hicieron posible una amplia difusión de estilos de baile identificado primeramente como tropical, pero con clara ascendencia cubana" (Juárez, 2011:171). En este sentido, tanto las "diosas y rumberas" exóticas que aparecían en los principales cabaret del país como los músicos integrantes de agrupaciones, estaban dando un sentido diverso a la variedad musical mexicana. Contrario a lo sucedido en México, los aportes a la música peruana se dieron a través de influencias en menor medida por los cubanos que visitaban escenarios limeños, siendo los intérpretes locales quienes se apropiaban de algunos de sus temas musicales reproduciéndolos localmente.

MIGRACIÓN DE CUBANOS AL PERÚ, FORMACIÓN DE COMUNIDADES EN PACHACAMAC O EL BARRIO DE LOS CUBANOS DE VILLA EL SALVADOR E INDICIOS DE LA SANTERÍA (19802000. PRIMERA ETAPA)

Es relevante mencionar la migración en masa de los cubanos a Perú como un hecho que no se vincula propiamente con la primera etapa de influencia musical cubana en el país, pero que años después, fue parte asidua en la extensión, masificación y difusión de la misma con la concentración de las comunidades de migrantes cubanos en Perú. Las primeras oleadas de movilización se originaron a partir de un proceso político migratorio relacionado con la Embajada de Perú en La Habana, situación que provocó una serie de posibilidades para los 10800 cubanos que se insertaron en dicho recinto aquel 4 de abril de 1980.Sin embargo, arribaron a suelo peruano solo 1800, otros se establecieron en Costa Rica y Estados Unidos (a través del Mariel).

Nosotros ingresamos en la Embajada de Perú en Cuba porque en ese momento era el país que estaba en problemas con Cuba y bueno Fidel quitó la custodia de esa Embajada y autorizó públicamente que toda persona que quisiera ingresar en esa Embajada pues podían ingresar $y$ pues entramos como 10.800 personas, después salimos en un avión rumbo a Costa Rica y estuvimos allí tres días y de allí nos trajeron acá al Perú. Pero ya no llegamos los 10,800 a Perú porque hubo un grupo que se quedó en Costa Rica, después vino lo del Mariel y mucha gente que se había quedado en la Embajada del Perú en Cuba se fue por el Mariel a Estados Unidos, acá llegamos como 1.800 nada más (Martha Suarez, entrevista, 09/05/2010).

La llegada a Lima provocó una serie de vicisitudes, entre estas el establecerse en el parque zonal "Túpac Amaru" del Distrito de San Luis, ubicado en la Avenida Aviación.Según algunos informantes, el gobierno les construyó dormitorios colectivos, además que se encargó de la alimentación, la salud y otras atenciones. Aún así, estos se vieron obligados a realizar diversas actividades remuneradas tales como, la venta de dulces, artesanías y comidas de origen cubano, las cuales distribuían en las principales avenidas y centros de la ciudad. Otros se dedicaron a realizar trabajos de albañilería, carpintería, herrería, jardinería, entre otros, algunas mujeres realizaban trabajo doméstico en casas particulares. No faltó quien se dedicara a la estafa, el robo y la prostitución, pues al poco tiempo, los cubanos se hicieron notar en el ámbito capitalino a través de los medios de comunicación que señalaban: "Muchos comercializan droga y son delincuentes incorregibles. Hasta se dijo que el monstruo de Pachacamac (famoso violador limeño) salió de la colonia cubana. Pero a pesar de la mala fama que tienen, muchos sudan la gota gorda...reparando licuadoras viejas $[y]$ jugando a adivinar el futuro con caracoles" (Menéndez y Rubio, 2005:2).

Entre los años de 1980-1983, los cubanos permanecieron en el Parque Zonal del Distrito de San Luis. De acuerdo a sus tradiciones religiosas como la santería, poco se rumoró sobre la práctica entre cubanos, salvo el antecedente de una santera a quien llamaban "mamá negra", quien fue muy popular entre los cubanos por 
su afiliación a la santería. Incluso algunos de los cubanos migrantes argumentaron que trajo sus "fundamentos" de Cuba, con los cuales hacía consultas y curaciones a otras personas en los recintos del parque zonal de San Luis. El resto de cubanos en su mayoría, no tenían fundamentos religiosos, es decir, no se habían sometido a procesos rituales en su lugar de origen, salvo un santero a quien reconocían como "obá" pero que no trabajaba la santería y dos espiritistas, quienes hacían limpias a algunos usuarios limeños con huevos y hierbas olorosas. Algunos de estos cubanos emigraron a otros países, entre ellos "mamá negra", se trasladaron principalmente a Brasil, Venezuela, Canadá y Australia. Durante el último año que permanecieron en el Parque Zonal, no se practicó la religión debido a la ausencia de fundamentos y elementos propios de la santería, ya que "...los santeros que quedaban no podían ejercer nada sin sus santos y nunca tuvieron la oportunidad de que alguien les trajera sus santos, entonces ellos no la ejercían tampoco, y babalaos acá no había... nos quedamos como huérfanos..." (Manuela, entrevista, 09/05/2010).

Después de permanecer tres años en el Parque Zonal del Distrito de San Luis, los cubanos se trasladan a la periferia de la ciudad en el Distrito de Villa el Salvador en la urbanización Pachacamac (Avenida Republicana, manzana "J", lote 31) motivados por los terrenos y casas de interés social que les construye el gobierno; según algunos informantes, fueron entre 600 y 800 personas quienes poblaron el conocido "barrio de los cubanos". Entre estos cubanos, también llegó la santera conocida como "mamá Rosa" (OloYemayá), quien se empieza a desempeñar como santera en el "barrio de los cubanos" y sus prácticas se popularizan entre los cubanos y después entre otros clientes limeños, a quienes les hacía "trabajos" como lectura

$3 \quad$ Se le conoce con esta jerarquía dentro de la santería a aquellos iniciados en la religión quienes ostentan el título de dirigentes de las ceremonias rituales dentro de la regla.

4 Oricha (deidad) representa el mar, se sincretiza dentro del pastoral judeocristiano con la Virgen de Regla. de caracoles y limpias, cobrándoles diez soles (tres dólares) por sesión. Cabe destacar que los rituales que realizaba no siempre aludían a la santería, sino a cultos locales como "baños florales"5, "pasar el huevo"6, "limpias con kuy"7, entre otros, los cuales realizó hasta su muerte en el año 2002. La santería en esta etapa se popularizó a través de la noción de "barrio", es decir, como una práctica que rescataba la tradición local, según algunos informantes, la casa de "mamá Rosa" era el centro de reunión para algunos grupos de cubanos quienes "jugaban dominó", "tomaban gaseosa" y "recordaban Cuba". Entonces, la noción de casa templo ya se distinguía como un espacio de interacción y a la vez, de reencuentro con la cultura local, el culto a los orichas y la noción de comunidad.

Esta noción de "barrio" vinculada a la santería se representó con la casa "religiosa" de "mamá Rosa", como el espacio que producía identidad, "el lugar" y a la vez, la ausencia de este en un espacio ajeno (cubanos en Lima).Si bien es cierto, en Cuba la casa templo es indispensable para los rituales religiosos, en Lima esta adquiere una doble importancia, es decir, no solo funge como casa religiosa, sino también como lugar de encuentro con la cultura de origen $y$ con el territorio imaginado. Para el caso de los limeños, la casa "religiosa" de "mamá Rosa", también cobró importancia, pues según Mercedes Álvarez"los clientes de Rosa la visitaban desde temprano y pasaban varias horas con ella, aún cuando no fueran a consultarse o a hacerse trabajos, la querían mucho, eran gentes [sic] de plata la mayoría y vivían en buenos lugares de Lima" (entrevista, 28/06/2010). Con el tiempo, la práctica de santería fue haciéndose visible a partir de las intenciones de algunos locales por incorporarse a la religión, "realizando" algunos rituales como el de "lavamiento

$5 \quad$ Ceremonias rituales pertenecientes al corolario esotérico, también se utiliza en la santería.

6 Ceremonia ritual usual en diversas tradiciones de carácter esotérico, pues se cree que aleja las energías negativas ocasionadas a las personas por malas voluntades.

7 Ceremonia ritual con animal endémico del Perú, mantiene características similares a la rata, pertenece a la familia de los roedores. 
de coco"8. Además, hacía algunos collares, los cuales "lavaba" con hierbas locales antes de entregárselos a sus clientes. También realizaba otros rituales de limpieza como el conocido "baño de río", en el cual limpiaba el cuerpo de las personas con gallinas y palomas, sacrificándolas después. Asimismo, enseñó a construir (montar) bóvedas espirituales en las casas particulares de sus clientes y los consultaba al estilo del espiritismo.

Después, "mamá Rosa" empezóa consultar con el diloggún y a realizar diversos tipos de trabajos como: "endulzamientos" o hechizos sentimentales, "despojos de energías negativas", "atracción de dinero" y "curaciones o barridas para el mal de ojo y la buena salud”. Según Mirtha O’Farril, “'mamá Rosa' se convirtió en santera aquí en el Perú, aquí empezó a practicar, todos los del barrio notábamos que solo trabajaba la ocha y tenía muchos clientes, además que empezó a vestirse de blanco y a colgarse los collares, a todos nos gustaba verla así porque nos imaginábamos que estábamos en Cuba..."9. Como Rosa estaba muy involucrada en la santería y tenía algunos clientes pertenecientes a estratos altos de Lima, pidió ayuda a algunos de sus "ahijados" para hacer llegar al país a un "ayudante" cubano con "conocimiento", el objetivo era el elaborar ceremonias rituales como el de YokoOcha ${ }^{10}$. Entre los años de 1990-1995, arribaron al país algunos santeros y espiritistas cubanos familiares $y$ amigos de Rosa, $y$ otros migrantes de la primera oleada, estos no se establecieron en Pachacamac, sino en otros distritos de Lima en los cuales "anclaron" sus templos y tuvieron sus propios ahijados. La entrada de cubanos al país se hizo recurrente a partir de la invitación que hacían los cubanos ya establecidos a otros que se encontraban en la isla. En esta etapa, se establecieron algunos cubanos quienes llegaron

8 Ceremonia ritual que realizaba "mamá Rosa” en comparación de la entrega de Elegguá.

9 Conversación con Mirtha O’Farril, emigrante cubana de la primera etapa, Villa el Salvador, 3 de junio de 2010.

Ceremonia jerárquica en la santería que consagra ritualmente al neófito en sacerdote. al país por competencias deportivas, campañas médicas, congresos académicos, entre otros eventos. No necesariamente arribaban a Perú, sino también lo hacían por otros países como Bolivia y Ecuador, para después "cruzar" la frontera e instalarse en Lima de forma definitiva.

\section{MIGRANTES CUBANOS, FORMACIÓN DE COMUNIDADES Y POPULARIZACIÓN DE LA SANTERÍA EN EL PERÚ (2000-2010. SEGUNDA ETAPA)}

La segunda etapa inicia después del año 2000 y hasta el 2010. La característica más relevante de dicha etapa es la popularización de la santería en la ciudad por limeños pertenecientes a estratos sociales medio/alto. Entre los cubanos migrantes también existen singularidades jerárquicas de carácter social, por ejemplo, hay casas templo que mantienen como miembros a algunos de los primeros anclajes (1980), otras solamente a cubanos que recientemente han emigrado al país. Las dos casas religiosas más populares en Lima se caracterizan por tener entre sus miembros a cubanos $y$ limeños que pertenecen a estratos altos en el país, sobre todo profesionales, personas públicas (futbolistas, actrices, presentadores de televisión, políticos). Los babalawos, santeros $y$ espiritistas que trabajan de forma "independiente" también han formado sus familias rituales, aunque estas representen minoría en comparación con otras pertenecientes a casas "legitimadas" como la de IlélfáOmí11. La mayoría de estos son independientes e interactúan entre sí, es decir, se apoyan en las elaboraciones de ceremonias rituales, en la adquisición de elementos religiosos y en las reuniones sociales (cumpleaños, festejos, otros). Regularmente no se vinculan para la organización del calendario ritual, salvo algunas fechas específicas que celebran de manera informal.

Un aspecto importante a resaltar en esta etapa, son las competencias por el "conocimiento" religioso entre cubanos. Estas luchas

$11 \quad$ Casa templo que se ha popularizado en Lima a partir del reconocimiento de usuarios locales por sus mentores cubanos. 
de poder, también se relacionan con la legitimación de las casas templo, ya que se encuentra en juego el estatus del babalawo. Regularmente cada casa crea polémica sobre otra, reconociendo la propia como única o en casos extremos, otras con las que han construido lazos de amistad, como narra Félix Calá:

...la única casa templo (en Lima) es la de Luisito, también esta una señora que se llama Alicia, también otra santera que es colombiana que se llama Angélica que tiene un programa de televisión, incluso esa es la señora más vieja, la santera más vieja aquí en el Perú, ella hizo todo en Cuba... yo soy el babalawo más viejo aquí ${ }^{12}$.

Otros líderes religiosos opinan lo contrario, argumentando que sus casas templo son las únicas consolidadas, desconociendo la existencia de otras casas religiosas. MidialaArgudín, santera de la casa IlélfáOmí, argumenta que:

...sí, hay varios babalawos, hay varias santeras y santeros —ihee! - sí, creo que sí, la familia religiosa de aquí está creciendo. Pero acá en Lima no conozco ninguna otra casa de santo, sé que como santeros y religiosos los cubanos hacen sus trabajos y ceremonias, pero que conozca otra casa de santo así como esta, no (Midiala, entrevista, 12/04/2010).

Otros líderes religiosos de la segunda etapa, reconocen que antes de su llegada a Lima, ya existían indicios de la santería transmitidos por los cubanos de Villa el Salvador, incluso algunos de estos miembros de la primera oleada se han vinculado con algunas casas templo como la de IlélfáOmí para recibir algunos fundamentos menores como guerreros, mano de Orula y Olókun, otros han coronado santo.

Acá en el año 1980 hubo un éxodo de personas cubanas y de ahí alguno que otro era religioso, no puedo asegurar ni

12 Observaciones de trabajo de campo, conversación con Félix Calá, Lima, Perú, 25 de junio de 2010. que haya habido santeros, ni que haya habido babalawos, según los que me explican algunas personas que conozco, había, creo, una santera o espiritista, uno que otro obá y uno que otro babalawo, no estoy clara en eso — - ihee!-De ese entonces hay dos o tres personas que de una manera de espiritismo han trabajado esta religión y la han introducido también acá, ya, al punto de que aquî hay peruanos que llevan como 8 o 9 años que tienen hecho santo, no sé si ha sido precisamente por este canal, con esta persona, o porque hayan ido a Cuba anteriormente $y$ hayan conocido a alguien. Me parece que hay un caso de eso, pero si hay por lo menos, hay peruanos con 8 o 10 años de santo (Midiala, entrevista, $12 / 04 / 2010)$.

Estas disputas entre religiosos son muy comunes, incluso entre miembros de la misma casa templo. Lo que sucede en Lima no es tanto una lucha por el conocimiento religioso, incluso ni por el estatus, sino más bien, por la posición de "encajar" en las clases altas, negando de esta forma, la existencia de otros $\mathrm{u}$ otras casas, haciendo referencia de la propia como única. Incluso, ofreciendo el contacto con otras casas templo fuera de Perú, en países como Cuba, México, Venezuela, Estados Unidos, entre otros. El posicionarse como líder de otras casas fuera del país es un reconocimiento que les ha sido útil a la mayoría de santeros y específicamente, a babalawos cubanos que residen en la ciudad. Estas estrategias de reconocimiento, también les ha facilitado la incorporación a diversas instituciones como: universidades, museos, la misma embajada de Cuba, entre otras, considerándolos como líderes religiosos, extendiéndoles invitaciones para impartir conferencias, charlas, cursos, etc. Además, se interesan en dirigir la Asociación Yoruba de Perú, aún cuando no se ha formado y los planes de integrarse como Asociación llevan varios años. La consolidación de la santería en Lima ha sido un proyecto que se han planteado algunos religiosos cubanos, pues según 
estos, les beneficiaría a todos el lograr afiliarla a la Asociación Yoruba de Cuba.

La migración de cubanos se extendió en esta segunda etapa, pues las invitaciones constantes se hicieron notar en los arribos masivos de los migrantes quienes ingresaban al Perú a través de la carta de invitación que enviaban amigos o familiares residentes en el país. Muchos de estos migrantes arribaron por diversas razones, a través de torneos deportivos en los cuales participaba la selección de Cuba, otros por los congresos de medicina en los cuales llegaban médicos cubanos regularmente como conferencistas. La mayoría de estos se insertó en el país a través de las agrupaciones de música que llegaban de Cuba para amenizar conciertos, sobre todo del género "salsa". Las comunidades de cubanos se formaron no en el barrio de los cubanos de Villa el Salvador, sino en diversos distritos de Lima. La popularización de la cubanidad se manifestó en las expresiones culturales encontradas en las noches habaneras de Lima, sobre todo en los lugares de ocio cubanos, restaurantes bares, discotecas y otros espacios que trataban de manifestar las identidades culturales caribeñas. Otro aspecto a considerar es la difusión de la comunidad cubana y sus tradiciones culturales a partir de páginas web, estaciones de radio, televisión y periódico en las cuales se hacía referencia a la popularización de la cubanía en Lima.

¡QUÉ VIVA CHANGÓ, QUÉ VIVA CHANGÓ! LA MÚSICA CUBANA Y EL FOLCLORE DE LA VIDA NOCTURNA EN LOS BARES DE MIRAFLORES COMO MEDIADORES DE LA SANTERÍA EN LIMA (1997-2010. SEGUNDA ETAPA MUSICAL)

¡Santa Bárbara bendita para ti surge mi vida, para ti surge mi vida y con emoción se inspira...qué viva Changó, qué viva Changó, que viva Changó, qué viva Changó señores. Con voluntad infinita arranco del corazón la melodiosa expresión pidiéndote desde el cielo nos envíes tu consuelo y tu santa bendición [coro] qué viva Changó...aché...cabiosilé...Virgen venerada y pura Santa Bárbara Bendita... yo también de corazón...haré que tu nombre suba y en el nombre de mi Cuba este saludo te envío...que viva Changó señores!(Celina y Routilio).

Esta es una estrofa de la canción titulada "Qué viva Changó" entonada por Celina yRoutilio en la década de los 40, considerada como una de las canciones cubanas más populares en su género al menos entre los limeños. La música fue un factor importante en la popularización de la santería en Lima, aunque en los años 80 los grupos musicales y cantantes que tuvieron auge fueron en su mayoría de Puerto Rico, Panamá, Venezuela y algunos cubanos, tales como Héctor Lavoe, Willie Colón, Rubén Blades, Óscar de León, Celina y Routilio, entre otros; para la década de los 90, aparecieron en los escenarios limeños grupos cubanos como "Elio Revé y su Charangón", "Manolito y su Trabuco", "Los Van Van", "Irakere", la "Sonora Matancera", la "Charanga Habanera", "Dan Den", entre otros, de los cuales resaltaron canciones como "Todavía nó", "Conga negro cana'o", "El temba y lola", "Cuéntamelo todo", "Somos cubanos", "Dile a Catalina", "Guantanamera", "Que viva Changó", etc.Es precisamente en esta oleada de grupos cubanos, de los cuales algunos de sus integrantes se establecen en Lima, tales como Dayron y Barbarita pertenecientes a la agrupación de los "Dan Den", entre otros, algunos de estos se dedicaron a la práctica de la santería de forma activa. Es interesante resaltar que la "salsa cubana" ha sido uno de los ritmos que ha gustado al colectivo limeño y el arraigo se debe a la popularización de los grupos de origen cubano que ya aparecían en los escenarios de Lima y El Callao; entre estos, algunos como "Los Van Van", la "Charanga Habanera" y Celia Cruz, los cuales entonaban ritmos y letras que aludían a los orichas, tales como "Soy todo", "Yemayá y Ochún", entre otros que hacían referencia a la religiosidad cubana.

La industria musical - tanto en Lima como en otros espacios del mundo- ha sido mediadora en el anclaje de cubanos y de la santería propiamente, ya que es a través de estos ritmos caribeños que hombres y mujeres limeños se relacionaron con la religión, además que se han construidoredes artísticasque han 
permitido que otros cubanos se establezcan en el país en calidad de invitados. Es relevante mencionar que esta etapa también se caracteriza por la vinculación entre la música y la religión como generadores de identidadesculturales entre las comunidades de cubanos y algunos limeños quienes se apropian de estas ideas religiosas. En México ha sucedido de manera similar, pues es a través de la música que muchos practicantes de santería se han familiarizado con la religión.

Mi conocimiento de la santería fue gracias a la música. Siempre he sido una gran amante de la música y los bailes cubanos y un día escuché la canción Soy todo de la mejor orquesta de timba que hay en Cuba, los Van Van. Esta canción habla de los orishas y del amor que el cantante le tiene a su religión y a sus raíces. Después de ello me dediqué a leer libros sobre santería, ir a tambores... (Ollin Islas Romo, entrevista, 29/01/2010).

A partir de los 90 con el arribo de los grupos musicales cubanos a los principales escenarios de Lima y el auge de la "salsa cubana", se establecieron diversos centros nocturnos al estilo cubano, actualmente se encuentran El Cohiba Club, El Tumbao, Aché Para Ti, Cohiba Café Cuban, entre otros;los cuales se han popularizado en la ciudad por la concurrencia de limeños y sobre todo, de la comunidad cubana quienes los consideran como "el rinconcito de Cuba en el pleno corazón de Miraflores".Estos establecimientos han sido el punto de encuentro de la comunidad cubana, sobre todo de la segunda oleada, es decir, de los que han llegado a partir del 2005, babalawos y santeros que se reúnen para gustar de los ritmos caribeños y recordar Cuba, ya que en estos también se ofrecen bebidas tales como, "Cuba libre", "mojitos", "daiquirí", "culto a la vida", etc.; gastronomías y lenguajes que se comparten a forma de "jerga" tales como, "asere", "consorte", "abbure", "aché", "iré" y entre estos, las conversaciones que aluden a lo religioso, los fundamentos recibidos, las jerarquías, los signos, los Orichas e Ifá. En estos espacios se tejen relaciones entre cubanos quienes se conocen y se distinguen a partir de la indumentaria religiosa como el portar "idé" (pulseras), "collares", "aros", así como, otros elementos que aluden a la santería. Del mismo modo, algunos locales se acercan para contactarlos $y$ pedirles consejos que en su mayoría terminan en consultas, eboses y hasta iniciaciones en la ocha (Saldívar, 2011 y 2012).

La música que se entona en dichos espacios ha sido un canal de propagación para los cultos de la santería, debido a que algunos limeños argumentaron sentirse atraídos por los ritmos y las letras que relacionaban a Cuba, su diáspora $y$ los orichas. De esta forma:

...la difusión que ha tenido la santería aquí se ha dado en un tanto por la música cubana que se ha expandido muchísimo...entonces a las personas les llama la atención todo esto, $y$ a pesar de la transculturación ha quedado vigente muchas cosas y nosotros nos identificamos con eso, con la música, con los cantos, y también con la obediencia, con lo espiritual (Luis García, entrevista, 07/09/2010).

En adelante, esta difusión se ha gestado a partir de una mayor presencia de cubanos en la ciudad, de bares, restaurantes y otras empresas que resaltan la cultura cubana en Lima, produciendo múltiples discursos en torno a los santos y los santeros "afamados", los cuales han legitimado la religión considerablemente. Este proceso de "filtración cultural" podría reconocerse como una vía de transnacionalización de símbolos religiosos que se establecen en diversos espacios del globo, entre estos, Lima. Así, la segunda etapa de transnacionalización de la santería culmina con el auge de la música $y$ las agrupaciones cubanas de salsa que arribaron a suelo limeño entre 1997 y el 2010, de las cuales, algunos de sus integrantes se anclaron en la ciudad convirtiéndose en empresarios y socios de restaurantes, bares y discotecas estilo cubano que se encuentran en su mayoría en el Distrito de Miraflores.

Cabe resaltar que muchos de los cantantes cubanos de la primera etapa se sintieron identificados con Perú, tal es el caso de Bienvenido Granda, Celia Cruz, Celio Gonzales 
y el dúo "Los Compadres", que se sentían tan cómodos en estas tierras como en su casa. Celio Gonzales fue sacado en hombros de la Plaza de Acho en los 50. Celia Cruz visitaba cada año Perú para las fiestas de fin de año en los 80 y principios de los 90, así como Lorenzo Hierrezuelo, del dúo "Los Compadres", tocaba sus interpretaciones con una guitarra peruana "Falcón". Llama la atención en el presente, cuando se escucha música del recuerdo en las emisoras radiales, suelen presentarla como "la música de los buenos tiempos", lo que evidencia que antaño, no solo las ejecuciones, la letra y la interpretación musical se relacionaban con las tradiciones culturales y la identidad, sino que también los sonidos hacían alusión a momentos épicos cotidianos. En este sentido, la migración de los cubanos a Perú en 1980, no trajo nada nuevo en el aspecto musical, los cubanos inmigrantes se fueron adaptando a la idiosincrasia peruana $y$ fue hasta mediados de los 90 que agrupaciones cubanas arribaron a escenarios limeños.

La segunda gran etapa de la música cubana empezó en Lima, sin lugar a dudas hacia 1997. El vals peruano agonizaba y sonaba recién un nuevo género "La tecnocumbia", término acuñado por la texana Selena Quintanilla y que en Perú fue un derivado de la cumbia andina, con elementos foráneos mexicanos y bolivianos. Este género fue importante porque, a criterio personal, comenzó a definir la identidad peruana. A fines de 1997, llegaron al país Willy Chirino y Albita Rodríguez, que hicieron un par de presentaciones en el Callao. En el verano de 1998, sonaba en la radio Isaac Delgado ("Loco" y "No me mires a los ojos"), "Manolito y su trabuco", "La Charanga Habanera" y Leo Vera. Sonaba en la radio "Los Van Van", "Carlos Manuel y su clan" y Adalberto Álvarez. Leo Vera, cantante que tuvo éxito con el tema "Hombre casado" de un disco de "Irakere", llegó a Lima para hacer algunas presentaciones; no obstante, pasó desapercibido. Generalmente, desde los 50, los intérpretes cubanos que llegaban solos contrataban orquestas con integrantes peruanos que conocían sin ningún problema todos sus temas.
La música cubana, esta vez "salsa" y timba, volvió a convocar a los limeños, una oleada de cubanos post 80 llegó a Perú en conjunto con jóvenes músicos que formaron "Son Café", base de los "Conquistadores de la salsa" y "Son Havana"; se relacionaron con el grupo peruano con influencia cubana "Camaguey" y "La progresiva del callao", pero aunque David Calzado y su Charanga Habanera grabaron un disco con la disquera popular "Rosita producciones" (2000), bajó la popularidad de la música cubana hacia el año 2001, siendo reemplazada por grupos locales de tecnocumbia y reggaetón. Del año 2000 al 2007, el apogeo de la música folklórica en Perú fue una total sorpresa, durante el 2005 llegaron los grupos cubanos "Dan Den" y "La Caroband", así como, retornaron "Los Van Van" y la "Charanga Habanera". Se creó en el distrito populoso de San Martín de Porres la famosa "Cubanada": grandes bailes con cerveza y música cubana de fondo. Luego de la muerte de los integrantes del grupo de cumbia "Néctar" en el 2007, en un accidente de tránsito en Argentina, volvió a Perú una segunda fiebre por la tecnocumbia ahora llamada solo cumbia. En esta época, muchos músicos cubanos se habían anclado en Perú, formando parte de la farándula limeña(Michel Maza, ex Charanga Habanera;Dayrón, Martín y Barbarita Fernández, ex Dan Den y el gallo de la salsa, ex Manolito y su trabuco), compartiendo escenario en muchos casos, con los intérpretes de la llamada "renovada cumbia"

La aparición de actores, cantantes y bailarines cubanos en la televisión, la formación de grupos peruanos de "salsa" y timba con integrantes cubano-peruanos como"Son Yemayá", "Team Cuba", "N`talla", "D'caratula", "D’farandula", "Mayimbe", "Ensamble", etc., así como, la llegada permanente de la "Charanga Habanera", "Los Van Van", "Elito Revé y su Charangón", creando un tercer apogeo de la música cubana. En el 2010, se llevó a cabo en el estadio de la Universidad Nacional de San Marcos el primer festival "Cubanísimo", repitiéndose con mayor éxito en el 2011, con notables figuras como "Elito Revé y su charangón", "Bamboleo", "Alexander Abreú y su Habana de Primera", "Los Van Van", "Maikel 
Blanco", "Pupi y su Son Son", "Giraldo Piloto y su Klimax"; por lo cual los organizadores acordaron que los festivales "cubanísimos" serían en los meses de diciembre de cada año. De esta forma, los viejos salseros de Puerto Rico, Nueva York, Panamá y Colombia se sorprendían por el arraigo de la salsa en un país sudamericano y andino como Perú, la sorpresa es hasta la actualidad mayor en los músicos cubanos que han experimentado los escenarios de espacios de ocio limeños como"Scencia", "Timbalero", "Voodoo", "Orula", "Achépa'ti", "Tumbao", "Cohiba" y otros con nombres alusivos a las religiosidades caribeñas, abarrotadas de público en sus distintas presentaciones.

\section{IDEAS PARA EL DEBATE}

Los estudios sobre migración internacional (Glick-Schiller, 1999; Guarnizo, 2005; Levitt, 2001 y 2003) han puesto seria atención en los procesos transculturales de las comunidades recolocadas, experiencias, conocimientos $y$ saberes que acompañan a los emigrantes durante sus travesías y también en los lugares de anclaje. En este sentido, se podría mencionar los procesos de transnacionalización como una representación a partir de la circulación de ideas, objetos materiales, tradiciones y otros elementos exportados a través de las comunidades que se establecen en diversos lugares (Hannerz, 1996; Perera, 2008 y Portes, 2003). Por su parte, los migrantes son "influidos por sus continuos lazos con su país de origen, o con las redes sociales que se extienden más allá de las fronteras nacionales" (Glick-Schiller y Levitt, 2004:61). Cuando se escucha el concepto "migración", rápidamente se piensa en algún lugar en específico, desde la perspectiva internacional, Estados Unidos ocupa un lugar importante en el epicentro de los movimientos físicos de las personas, aunque también se consideran otros casos como la migración de peruanos a Chile; de bolivianos y paraguayos a Argentina y Brasil; de mexicanos, cubanos y puertorriqueños por nombrar algunos- a los Estados Unidos, entre otros. Pero, ¿acaso se ha pensado o estudiado en términos científicos, la migración de cubanos a Perú y sus escalas recurrentes?, en lo personal, no se ha encontrado estudios recientes que contesten la pregunta.

La migración de cubanos a Perú no es un fenómeno nuevo, sino que mantiene antecedentes históricos que van más allá de los 30 años, por tanto sepuede cuestionar lo siguiente ¿cómo se representa la cultura de origen (local) en el lugar donde se establecen los migrantes? Un ejemplo interesante para matizar estos movimientos migratorios se puede notar en las relaciones migratorias de peruanos a Chile. Carolina Stefoni menciona cómo durante la década de los 90, la formación de comunidades de peruanos en el centro de Santiago, se originó a partir de un número reducido de estos quienes "lograban reunirse al costado de la Catedral o en algún restaurante chileno, a compartir información y experiencias vividas en Santiago $y$ recordar con cierta nostalgia a todo lo que había quedado en Perú" (2004: 2). La misma autora argumenta que después de un tiempo, las comunidades formaron sus propios espacios a partir de la construcción de bares, discotecas y restaurantes al estilo peruano, servicio de envío de encomienda (Ibid.), transformando parte de la imagen de la ciudad y convertirla en lo que se conoce como "Lima chica" (Luque, 2003).

En este mismo sentido, Jorge Duany hace referencia a la migración de puertorriqueños a Estados Unidos. El autor menciona que el país se ha convertido en:

...una nación en vaivén: un país cuyos bordes son cruzados incesantemente por migrantes que van $y$ vienen... Al mismo tiempo, Puerto Rico ha recibido a cientos de miles de inmigrantes... de retorno $y$ sus descendientes, así como ciudadanos de otros países, sobre todo la República Dominicana y Cuba (2001:57).

La metáfora que propone el autor como "nación en vaivén", explica no solo los desplazamientos de locales al exterior, ni de otros lugares al interior, sino la forma en la cual se re-construyen ciertos símbolos culturales a partir de las interacciones sociales, procesos que se hacen visibles en la identidad nacional. Uno de los ejemplos latentes es la consideración de los mismos puertorriqueños en Estados 
Unidos sobre todo de la diáspora establecida en New York como newyorican, migrantes circulares que siguen perteneciendo a una nación en específico pero que han adoptado otras identidades relacionadas al lugar donde residen. Esta representación de puertoriqueñeidad se hace evidente "en las escuelas de Brooklyn, los festivales folklóricos del Parque Central, el Desfile Puertorriqueño a lo largo de la Quinta Avenida de Nueva York y las exhibiciones etnográficas en el venerable museo del Smithsonian Washington, DC" (2001: 62-63).

Steigenga, Palma y Girón destacan cómo el transnacionalismo para la comunidad de guatemaltecos que radican en Júpiter, Florida, se representa a través de "los grupos sociales, las identidades, las creencias, los rituales, las prácticas y las relaciones de poder en las comunidades de origen y las de destino" (2008:2), proceso por el cual "los migrantes están insertos en redes que se extienden a lo largo de múltiples estados y que las identidades, así como la producción cultural de los migrantes, reflejan sus múltiples localidades" (Glick-Schiller y Levitt, 2004:64). En este sentido, los autores plantean cómo la comunidad maya de Guatemala en Júpiter, Florida, no solo ha territorializado el lugar, sino también ha re-construido parte de la cultura local a través de la producción de símbolos religiosos, gastronómicos, lingüísticos, etc., insertando las prácticas locales en contextos un tanto diversificados. En este caso:

...los mayas en Júpiter... abarcan elementos físicos, culturales, ideológicos, familiares, religiosos, nacionales y locales que se combinan para crear un sentido de pertenencia e identidad en un ambiente confuso... la celebración de la fiesta, la organización de torneos de fútbol... la presencia de la marimba en eventos comunitarios, etc. (Ibid., 2008:7).

Es interesante resaltar cómo las diásporas de cubanos que se establecen en espacios determinados, después de un tiempo, al adquirir la ciudadanía, se convierten en transmigrantes (Levitt, 2003); es decir, personas que se encuentran viajando constantemente entre el país de residencia y origen.Además de viajar a sus países de origen, también lo hacen a otros lugares en los cuales pretenden formar casas templo, buscando como estrategia primordial, la ciudadanía del país en el que desean establecerse. Para el caso peruano, es importante señalar que la mayoría de los santeros y babalawos han buscado otros espacios como México, Venezuela o Estados Unidos, con objetivos de formar casas templo y familias rituales. En ocasiones estas casas religiosas no dependen solo del "padrino" cubano, sino también de otros "ahijados" babalawos locales quienes muchas veces se responsabilizan de la casa templo formada por el primero. De esta forma, la santería se convierte en una "religión red", dependiente siempre de la matriz cubana como legitimadora de las prácticas.

Es de interés mencionar la noción de familia, barrio, religión y nacionalidad que producen las prácticas de la santería, vinculadas desde el momento en el cual los grupos se desplazan a otros espacios y se relacionan con otros contextos (personas, países y lugares), donde las prácticas religiosas, a la vez, son prácticas que resaltan la identidad nacional (ritmos musicales, discursos afines, etc.) y la casa templo más allá de ser un espacio religioso, es un lugar donde se reúnen para festejar, conversar y reconocer el patrimonio existente, la ausencia del otro y la identidad nacional. Para el caso específico de los migrantes cubanos practicantes de santería que radican en Lima, es notable cómo estos al paso del tiempo se convierten en empresarios, es decir, que más allá de mercantilizar las prácticas de la santería y la formación de centros botánicos, también incursionan en la venta de ropa, de libros y hasta de productos gastronómicos, de los cuales transportan a Cuba, incluso forman pequeñas empresas en sus ciudades de origen $y$ a la vez, en el lugar donde radican. Por ejemplo, para el caso peruano la mayoría de practicantes de santería (dedicados exclusivamente a la religión) que han obtenido la residencia y pueden viajar a Cuba en calidad de turistas, han iniciado "pequeños" negocios de ropa, artículos de cocina, herramientas automotrices, entre otros. De regreso, introducen elementos religiosos, además de envíos que le hacen amigos y 
familiares a cubanos que residen en Lima y no tienen acceso a la isla. Esta forma de emprender negocios, también es una estrategia para estar en contacto con la cultura local, visitar amigos, familiares y recrearse del patrimonio local.

\section{CONCLUSIÓN}

La migración de la diáspora cubana a Perú ha sido poco estudiada, sobre todo en su estructura sociocultural, es decir, analizando fenómenos periféricos como la música y la religión. Estos movimientos transnacionales de personas, saberes y exotismos diversos, han construido múltiples identidades vinculadas con las comunidades de migrantes (quienes dejan su país de origen y se establecen de forma definitiva en otro lugar), los locales (quienes frecuentan a las comunidades de cubanos y se familiarizan con las costumbres propias) y la sociedad en general (quienes adoptan gustos musicales, recurren espacios de ocio cubanos como: bares, discotecas, restaurantes y otros). La música ha sido un canal de importante presencia para la comunidad cubana en el país, principalmente en Lima, ya que estos ritmos ofrecen paisajes culturales que enaltecen a Cuba y su diáspora. La religión por su parte, ha posibilitado gestar una serie de códigos culturales que se interconectan con las tradiciones locales a partir de la puesta en escena de ritos, mitos y leyendas ancestrales practicadas por familias rituales peruanas. Finalmente, se puede notar cómo esta amalgama de significados, ha construido puentes políticos, económicos y culturales a partir del anclaje de símbolos transnacionales en sus procesos diversos de asentamiento, construcción y popularización entre personas, países y lugares.

\section{BIBLIOGRAFÍA}

\section{LIBROS}

Chamorro, Arturo. Los instrumentos de percusión en México. Zamora, México: El Colegio de Michoacán-Conacyt, 1984.

Figueroa Hernández, Rafael. "Rumberos y jarochos". La Habana/Veracruz, Veracruz/La Habana. Las dos orillas.
Bernardo García Díaz y Sergio Guerra Vilaboy (eds.). Xalapa, México, Universidad Veracruzana y La Habana, Cuba, Universidad de La Habana.2002: 383-399.

Flores y Escalante, Jesús. Historia documental y gráfica del danzón en México: Salón México. México: Asociación Mexicana de Estudios Fonográficos, 2006.

Glick-Schiller, Nina. "Transmigrants and nation-states: something old and something new in US immigrant experience". Handbook of international migration: the American experience. Charles Hirschman, Philip Kasinitz y Josh DeWind(eds.). New York, Eeuu. Russell Sage Foundation,1999:94-119.

Hannerz, Ulf. Conexionestransnacionales. Personas, gente, lugares. Madrid, España: Ediciones Cátedra, 1996.

Juárez, Nahayeilli. "Lo "afro" en las industrias de la música y el cine: el caso afrocubano en México". Circulaciones culturales. Lo afrocaribeño entre Cartagena, Veracruz y La Habana. Freddy Ávila Domínguez, Ricardo Pérez Montfort y Cristian Rinaudo (eds.). México: Publicación de la Casa Chata, 2011.

Knauer, Lisa. "Afrocubanidadtranslocal: la rumba y la santería en New York y La Habana”. Culturas encontradas: Cuba y Estados Unidos. Rafael Hernández y John H. Coastworth (eds.).Massachusetts, EEuu.David Rockefeller Center forLatin American Studies-Drclasde la Universidad de Harvard,2001: 11-31.

Monsiváis, Carlos. "El cine nacional". Historia General de México. El Colegio de México (ed.).México.co:racruzana y La Habana, Cuba: El Colegio de México-Harla,1988: 1506-1531.

Perera, Ana. "Redes transnacionales, representaciones sociales y discursos religiosos en Cuba".América Latina $y$ el Caribe. Territorios religiosos $y$ desafíos para el dialogo.Aurelio Alonso (compilador). Buenos Aires. Consejo Latinoamericano de Ciencias Socialesclacso,2008: 163-199. 
Sevilla, Amparo. "Los salones de baile: espacios de ritualización urbana". Cultura y comunicación en la ciudad de México. La ciudad y los ciudadanos imaginados por los medios. Néstor García Canclini (coord.). México.Universidad Autónoma de México (unam) Grijalbo,1998: 220-226.

Yúdice, George. "La industria de la música en el marco de la integración América Latina - Estados Unidos”. Integración económica e industrias culturales en América Latina. Néstor García Canclini y Carlos Moneta (eds.).México. Grijalbo,1999: 115-161.

\section{PUBLICACIONESPERIÓDICAS}

Duany Jorge. "Irse pa' fuera": los modos de vida móviles de los migrantes circulares entre PuertoRico y los Estados Unidos". Temas: cultura, ideología, sociedad 26. La Habana, Cuba,2001: 1-18.

Levitt, Peggy. "Transnational migration: taking stock and future directions". Global Networks 1(3). Wiley-Blackwell, 2001.

Levitt, Peggy. "You know, Abraham really was the first immigrant: religion and transnational migration".International Migration Review 37.New York, EeuU. Center for Migration Studies, 2003: 847-873.

Levitt, Peggy y Glick-Schiller, Nina. "Perspectivas internacionales sobre migración, conceptual la simultaneidad". Migración y desarrollo3. Zacatecas, México.Red Internacional de Migración y Desarrollo, 2004: 60-91.

Podalsky, Laura. "Guajiras, mulatas y puros cubanos: identidades nacionales en el cine prerevolucionario". Archivos de la Filmoteca 31.Valencia, España. Instituto Valenciano de Cinematografía,1999: 156-171.

Portes, Alejandro. "Conclusión: theoretical convergenciesand empirical evidence in the study of immigrant transnationalism”. International
Migration Review37. New York, Eeuu. Center for Migration Studies,2003: 874-892.

Saldívar, Juan. "El desarrollo del turismo religioso translocal. El caso de la santería afrocubana en Lima, Perú". Reflexiones 1 (91). San José, Costa Rica. Editorial de la Universidad de Costa Rica, 2012: 127-138.

Steigenga, Palma y Girón, Carol. "El transnacionalismo y la movilización colectiva de la comunidad maya en Júpiter, Florida. Ambigüedades en la identidad transnacional y la religión vivida”. Migraciones Internacionales 4(4). México.El Colegio de la Frontera Norte, AC, 2008: 38-71.

TESIS

Medone, Gloria. "Ensayo para la formulación de una teoría analítica de la interpretación musical sobre criterios de inteligibilidad. Concepción y construcción de índices e indicadores para un examen críticoobjetivo de la interpretación". [Tesis de Doctorado en Arte y Musicología]. Oviedo, España. Universidad de Oviedo, 2011.

Saldívar, Juan. "Un silbato para Elegguá. La producción transnacional de la santería en la ciudad de Lima”. [Tesis de Maestría en Antropología Social]. Perú:Pontificia Universidad Católica del Perú, 2011.

\section{TEXTOS ELECTRÓNICOS}

Menéndez, Ronaldo y Rubio Desirée. "El éxodo del Mariel, 25 años después: crónica de cuatro vidas". Nota periodística virtual. Madrid, 21 de setiembre de 2005. En: $<$ http://www.amigosdevilla.it/cuentos/ relato006.htm $>$ [Consultado el 30/06/2015].

Stefoni, Carolina. Inmigrantes transnacionales: la formación de comunidades $y$ la transformación en ciudadanos. 
Santiago, Chile. Flacso, 2004. En:<http:// bibliotecavirtual.clacso.org.ar/ar/libros/ chile/flacso/artstef.pdf $>$ [consultado el23 de febrero de 2011].

\section{ENTREVISTAS}

José (seudónimo).Ex trabajador marítimo de Puerto del Callao durante los años 19361973. Lima, Perú.10 de noviembre de 2010.

Luis García.Santero cubano emigrante de la segunda oleada. Lima, Perú. 07 de setiembre de 2010 .

Martha Suarez.Emigrante cubana de la primera etapa. Lima, Perú.09 de mayo de 2010.

Manuela Pérez (seudónimo). Santera cubana emigrante de la primera etapa. Lima, Perú. 09 de mayo de 2010.

Mercedes Álvarez. Santera cubana emigrante de la primera etapa. Lima Perú. 28 de junio de 2010 .

Midiala. Santera cubana emigrante de segunda etapa. Lima, Perú. 12 de abril de 2010.
Ollin Islas Romero.Santera mexicana. Méxicodf, México.29 de enero de 2010.

OTROS DOCUMENTOS

Guarnizo, Luis. "El estado y la migración global colombiana”. Ponencia. Segunda Conferencia Internacional sobre Relaciones Estado-Diáspora. Ciudad de México, México,2005.

Luque, José. "Transnacionalismo político. Identidad nacional y enclave étnico. El caso de los inmigrantes peruanos en Santiago de Chile". Ponencia. II Congreso Latinoamericano de Ciencia Política. Asociación Latinoamericana de Ciencia Política (alacip).Ciudad de México,México,2003.

Fecha de ingreso: 25/ 04/2014 Fecha de aprobación: 30/09/2014 
